\title{
September 2014 Pulmonary Case of the Month: A Case for Biblical Scholars
}

\author{
Lewis J. Wesselius, MD \\ Department of Pulmonary Medicine \\ Mayo Clinic Arizona \\ Scottsdale, AZ
}

\section{History of Present IIIness}

A 66-year-old man was seen in consultation. He had been followed since 1998 for bronchiectasis. He had a prior history of multiple skin infections with abscess formation requiring drainage beginning when he was in his 20 's. He presented with increased recent sputum production, greenish in color.

\section{$\mathrm{PMH}, \mathrm{FH}, \mathrm{SH}$}

He had a history of multiple skin infections, multiple pneumonias and osteomyelitis in addition to the bronchiectasis. There was a positive family history of coronary artery disease and childhood cancer in a sister. He had smoked cigars in the remote past, but none since the age of 25 .

\section{Physical Examination}

General: short stature, scoliosis, SpO2 98\% on RA

Chest: few scattered crackles, no wheezes

Cardiovascular: regular rate and rhythm with no murmur noted

Extremities: No clubbing, cyanosis or edema.

\section{Spirometry}

FVC $69 \%$ of predicted; FEV1 $76 \%$ of predicted.

Which of the following should be performed at this time?

1. Immunocompromised evaluation

2. Sputum culture

3. Thoracic CT scan

4. 1 and 3

5. All of the above 


\section{Correct! \\ 5. All of the above}

For evaluation of bronchiectasis, thoracic CT scan is usually considered the procedure of choice. The patient's CT scan is shown in Figure 1.

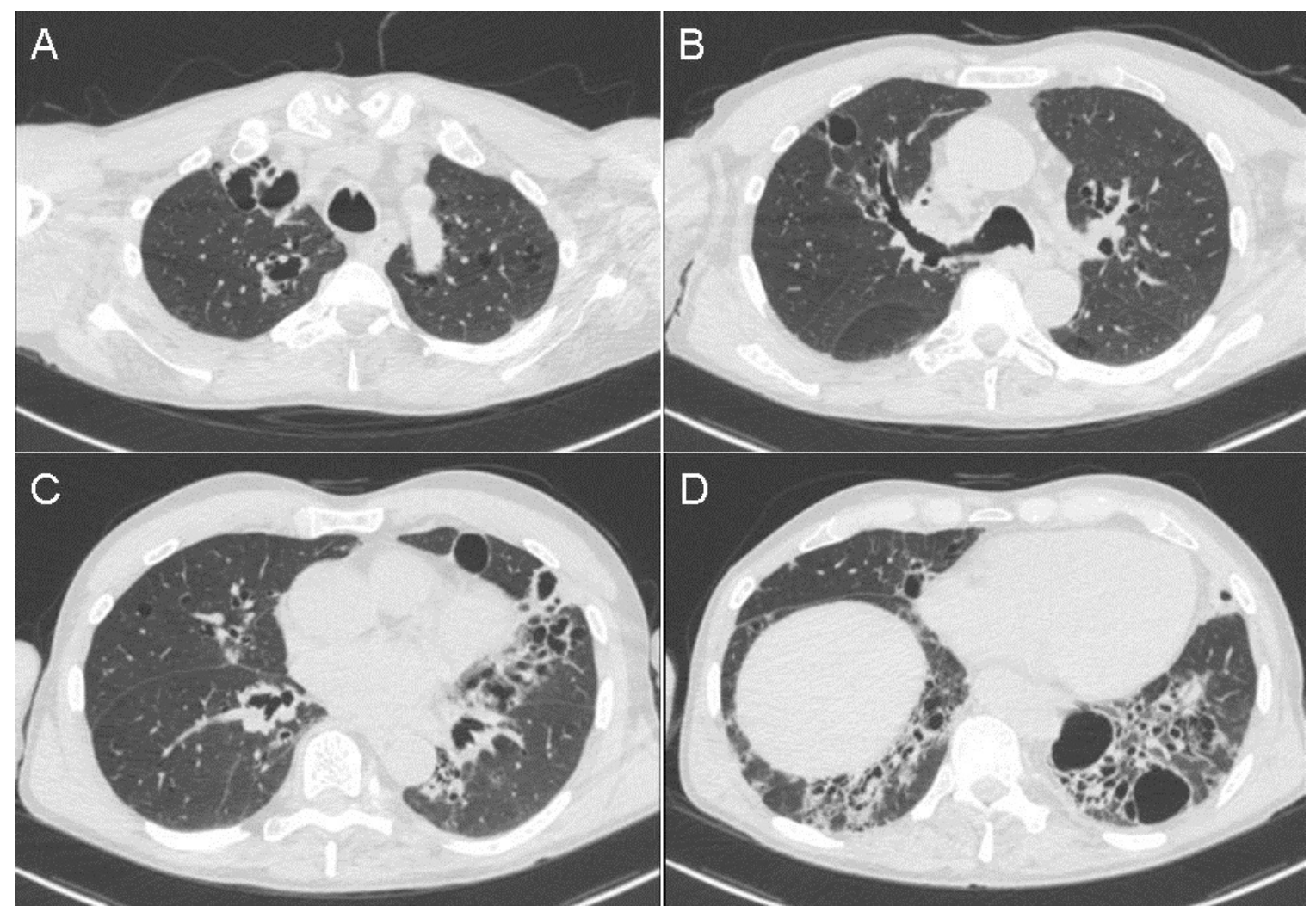

Figure 1. Representative images in lung windows from thoracic CT scan.

Sputum culture showed 3+ Pseudomonas aeruginosa (mucoid) and Mycobacterium avium intracullare (MAI).

The multiple skin infections and repeated pneumonias suggest the possibility of immunocompromise. HIV was negative. Immunglobulins showed an unremarkable $\mathrm{IgG}$ and $\mathrm{IgM}$ but a markedly elevated $\mathrm{IgE}$ of $7419 \mathrm{kJU} / \mathrm{mL}$ (normal $<380 \mathrm{kJU} / \mathrm{mL}$ ).

Which of the following disease(s) is/are associated with elevated IgE?

1. Allergic bronchopulmonary aspergillosis (ABPA)

2. Cystic fibrosis

3. Hodgkin's disease

4. Valley fever

5. All of the above 


\section{Correct!}

\section{All of the above}

A number of diseases have been associated with elevated IgE levels and can be broadly grouped as below in Table 1 .

Table 1. Disorders associated with elevated IgE levels Infections: Parasitic, HIV, Mycobacterium Tb, fungal (cocci), CMV Atopic Disease: Asthma, ABPA, Atopic Dermatitis Immunodeficiencies: Hyperimmunoglobulin E syndrome, Wiskott-Aldrich Syndrome Inflammatory Disease: Churg-Strauss, Kimura disease Neoplasms: Hodgkin's lymphoma, IgE myeloma Other: Cystic fibrosis, nephrotic syndrome

Our patient had no evidence of asthma and a sweat chloride for cystic fibrosis was negative.

Which of the following is the likely cause of his elevated lqEE?

1. Hyperimmunogloublin $E$ syndrome

2. IgE myeloma

3. Kimura disease

4. Strongyloidiasis

5. Wiskott-Aldrich syndrome 


\section{Correct! \\ 1. Hyperimmunoglobulin E Syndrome}

There is no evidence of myeloma in this patient. Kimura disease is a disorder of unknown etiology associated with lymphadenopathy. Strongyloidiasis is a chronic infection with the roundworm often seen in the Southeast United States. WiskottAldrich syndrome is a rare X-linked recessive disease characterized by eczema, thrombocytopenia, immune deficiency, and bloody diarrhea (secondary to the thrombocytopenia).

Hyperimmunoglobulin E syndrome (HIES) is also know as Job's syndrome. The name Job's syndrome is taken from the following bible passage:

"So went Satan forth from the presence of the LORD and smote Job with sore boils from the sole of his foot unto his crown." - King James Bible

In 1966 HIES was first described and characterized by eczematoid dermatitis and recurrent skin and pulmonary infections (1). In 1972 the association with elevated IgE was first appreciated. Clinical features of HIES include the following:

- Eczema - $100 \%$

- Peak serum $\lg \mathrm{E}>2,000 \mathrm{kJU} / \mathrm{mL}-97 \%$

- Eosinophilia - 93\%

- Boils $-87 \%$

- Recurrent pneumonias $-87 \%$

- Characteristic facies - (prominent forehead, facial asymmetry) - 83\%

- Scoliosis - 63\%

- Coronary aneurysms $-37 \%$

Which of the following signal transduction pathways has been identified as the cause of HIES?

1. G Protein-coupled signal transduction

2. RAS-mitogen activated protein kinase signal transduction

3. STAT3 signal transduction

4. Transforming growth factor- $\beta$ (TGF- $\beta$ ) signal transduction

5. Toll-like receptor TLR3 signal transduction 


\section{Correct! \\ 3. STAT3 signal transduction}

In 2007 an autosomal dominant mutation in signal transducer and activator of transcription factor 3 (STAT3) was identified as the link for infections and connective tissue abnormalities. STAT3 is integral to signal transduction for multiple cytokines (some pro-inflammatory, some anti-inflammatory) and mutations in STAT3 are generally regarded as the cause of HIES (1). Abnormalities in STAT3 result in failure of Th17 CD4 cell differentiation and a defect in neutrophil migration (chemotaxis) contributing to the increased risk of infection.

Patients with HIES usually present with skin infections/skin rashes early in life; mucocutaneous candidiasis; recurrent sinopulmonary infections with aberrant healing; bronchiectasis; and pneumatoceles. There is an increased risk of malignancies - most often non-Hodgkin's lymphoma, but others have been reported. There is also an increased incidence of vascular abnormalities.

Treatment of HIES includes proper skin care; aggressive treatment of infections; and prophylactic antibiotics may be appropriate in some patients (1). Bone marrow transplantation has been performed in a few patients with improvement reported (1).

\section{Reference}

1. Sowerwine KJ, Holland SM, Freeman AF. Hyper-lgE syndrome update. Ann NY Acad Sci. 2012;1250:25-32. [CrossRef] [PubMed] 